\title{
The Rodent Lumbar Spinal Cord Learns to Correct Errors in Hindlimb Coordination Caused by Viscous Force Perturbations during Stepping
}

\author{
Chad Heng ${ }^{1}$ and Ray D. de Leon ${ }^{2}$ \\ ${ }^{1}$ Department of Biological Science and ${ }^{2}$ School of Kinesiology and Nutritional Science, California State University, Los Angeles, California 90032
}

\begin{abstract}
The nervous system can adapt to external forces that perturb locomotion by correcting errors in limb movements. It is believed that supraspinal structures mediate these adaptations, whereas the spinal cord contributes only reflexive responses to perturbations. We examined whether the lumbar spinal cord in postnatal day 5 neonatal spinally transected (ST) rats corrected errors in hindlimb coordination through repetitive exposure to an external perturbation. A robotic device was used to deliver a viscous (velocity-dependent) force that opposed only the forward movement of the ankle in one hindlimb while the ST rats performed hindlimb stepping on a treadmill. We measured the interval between paw contact in the perturbed hindlimb and toe off in the unperturbed hindlimb. Before the force was activated, a normal pattern of coordination occurred: paw contact in the perturbed hindlimb occurred before toe off in the unperturbed hindlimb. This sequence was initially disrupted when the force was activated and the unperturbed hindlimb initiated swing during the swing phase of the perturbed hindlimb. Within five step cycles of exposure to the unilateral viscous force, however, the ST rats regained the preforce pattern of hindlimb coordination. These findings suggest that in the absence of supraspinal input, the lumbar spinal circuitry is capable of processing a complex ensemble of sensory information to maintain locomotor stability. Thus, the lumbar spinal circuitry may play a greater role in generating locomotor adaptations than previously thought.
\end{abstract}

Key words: rat; adaptation; transection; treadmill; stepping; robot

\section{Introduction}

When locomotion is unexpectedly perturbed, the nervous system adjusts limb movements in response to the perturbation. Some responses are acquired through practice, whereas other responses are more reflexive in nature. It is believed that the spinal cord contributes to locomotor adaptations by generating rapid responses to perturbations. For example, Forssberg (1979) and colleagues demonstrated that when spinally transected (ST) cats walked on a treadmill, they generated a "stumbling corrective reaction" to step over an obstacle. When the dorsum of the hindpaw came into contact with the blocking object, spinal reflexes were elicited causing an enhanced lift of the hindpaw. These results and other results (Timoszyk et al., 2002) indicated that the lumbar spinal cord generated fast corrective responses during stepping based on sensory feedback from the hindlimbs.

The question we asked in the present study was whether the lumbar spinal cord was also capable of learning to respond to locomotor perturbations. In contrast to the fast responses that were reflexively elicited by the perturbation, a learned response

\footnotetext{
Received April 11, 2007; revised June 26, 2007; accepted June 28, 2007.

This work was supported by National Institutes of Health Grant NS 42951-01S. We thank Kevin Vallance for his technical contributions to this study.

Correspondence should be addressed to Dr. Ray D. de Leon, Associate Professor, Department of Kinesiology and Nutritional Science, California State University, Los Angeles, 5151 State University Drive, Los Angeles, CA 90032 8162. E-mail: rdeleon@calstatela.edu.

DOI:10.1523/JNEUROSCI.1635-07.2007

Copyright $\odot 2007$ Society for Neuroscience $\quad$ 0270-6474/07/278558-05\$15.00/0
}

would require practice and thus learning would occur after some exposure to a perturbation. Recent studies have shown that humans learn to adjust stepping patterns after experiencing several step cycles of perturbations caused by a splitbelt treadmill (Morton and Bastian, 2006) and by robotic forces that resist leg movements during treadmill stepping (Emken and Reinkensmeyer, 2005; Lam et al., 2006). There is evidence that the cerebellum plays an important role in locomotor adaptations that are acquired through practice (Morton and Bastian, 2006). To study spinally mediated locomotor adaptations, we used a robotic device to deliver a viscous (i.e., velocity-dependent) force that opposed forward movement of the ankle in one hindlimb while ST rats stepped on a treadmill. We found that the unilateral viscous force initially perturbed hindlimb coordination, but the ST rats adjusted hindlimb coordination patterns within five perturbed steps. These findings suggest that in the absence of any input from supraspinal structures, the lumbar spinal cord is capable of correcting errors in hindlimb coordination through practice.

\section{Materials and Methods}

Experimental design. Twenty-eight female Sprague Dawley rat pups received a complete spinal cord transection at a mid-thoracic level $5 \mathrm{~d}$ after birth (Cha et al., 2007). Neonatal transected rats were used instead of adult transected rats because neonatal, but not adult, ST rats spontaneously recover hindlimb stepping, thus facilitating the locomotor tests. After the pups reached an adult age $(>30 \mathrm{~d}$ old), preliminary tests of hindlimb treadmill stepping were performed using a robotic device. Ten of the 28 ST rats performed consistent hindlimb treadmill stepping while 
in the robotic device (minimum of 20 consecutive step cycles) and were selected for the experiments. The remaining rats were not used because they stumbled frequently, making them inappropriate for tests of locomotor perturbations. Although treadmill training would have likely improved stepping consistency (Cha et al., 2007), we chose not to train the ST rats because the robotic perturbation may have been less effective in inducing coordination errors in trained ST rats. During the experiments, the ST rats first performed 10 steps without any forces applied to the hindlimb (i.e., preforce condition). Then, a unilateral viscous force was delivered by one of the arms of the robotic device to oppose the forward movements in one ankle for 10 steps. After the 10th perturbed step, the force was inactivated, and the rats performed 10 additional steps in the absence of the perturbation (i.e., postforce condition). In addition to the viscous force testing, the rats were tested under a null force condition using the same protocol. The tests of stepping with the null force were performed before the viscous force tests. Movements of the ankle were recorded by the robotic device for subsequent kinematic analyses. All procedures were approved by the Institutional Animal Care and Use Committee at California State University, Los Angeles.

Locomotor testing with viscous force perturbations. A robotic device was used (Rodent Robotic Motor Performance System; Robomedica, Irvine, CA) to test treadmill stepping and to apply the perturbing forces during stepping. The robotic device has been described previously (Timoszyk et al., 2002) and consists of two lightweight robotic arms, a body-weight support system, and a variable-speed treadmill. The robotic arms attach to the ankles of the hindlimbs via small loops of neoprene that wrap around the ankle. A computer-controlled, spring-actuated body-weight support system controlled the amount of weight bearing on the hindlimbs. Rats are placed in a harness that is secured to the body-weight support system using Velcro. All locomotor tests were performed at a treadmill speed of $10 \mathrm{~cm} \cdot \mathrm{s}^{-1}$ while the robotic device supported $85-90 \%$ of the rat's body weight.

To perturb stepping, a force was applied at the lower shank that was linearly related to the velocity of the ankle:

$$
\left(\begin{array}{l}
F_{x} \\
F_{y}
\end{array}\right)=\left(\begin{array}{ll}
B_{x x} & B_{x y} \\
B_{y x} & B_{y y}
\end{array}\right) \times\left(\begin{array}{l}
\dot{x} \\
\dot{y}
\end{array}\right)
$$

where $F_{x}$ and $F_{y}$ are the viscous forces in the $x$ and $y$ directions, respectively; $B_{i i}$ are the viscous field coefficients; and $\dot{x}$ and $\dot{y}$ are the $x$ and $y$ direction velocities, respectively.

The force was applied during the swing phase, which is defined by positive horizontal velocity. The horizontal viscous field coefficient, $B_{x x}$, was equal to $-0.24 \mathrm{~N} / \mathrm{m}$. The values for the other viscous field coefficients $\left(B_{y y}, B_{x y}\right.$, and $\left.B_{y x}\right)$ were set to zero. This is consistent with applying a force during swing, and the force is applied in the opposite direction to the horizontal velocity.

Data collection and analyses. Ankle movements during all tests were recorded by the robotic arms at $500 \mathrm{~Hz}$ and stored on a computer for subsequent kinematic analyses as described previously (Timoszyk et al., 2002). Briefly, the beginning of swing [toe off (TO)] and stance [paw contact (PC)] was identified based on changes in horizontal velocity. Once these events were detected, the interval of time between PC in the perturbed hindlimb and TO in the unperturbed hindlimb (PC-TO interval) and additional step cycle characteristics (e.g., length, height, duration) were calculated. A repeated-measures ANOVA with post hoc test (Bonferroni method) was used for statistical analyses of all kinematic variables (SPSS 13.0 software; SPSS, Chicago, IL). Data from all 10 of the rats were used for the statistical comparisons between the preforce and viscous force conditions. However, only four rats completed the postforce condition testing without stumbling. Analyses of the postforce condition were therefore based only on data collected from these four rats. We also measured the amount of time that the hindpaw was suspended over the treadmill belt during swing. Hang-time calculation was based on the vertical velocity of the hindpaw during swing. Specifically, the length of time in which the vertical velocity was close to 0 (i.e., between 25 and $-25 \mathrm{~mm} \cdot \mathrm{s}^{-1}$ ) was measured. We then examined whether hang time during swing was correlated to the length of the PC-TO interval. A correlation analysis was performed using the Pearson correlation.

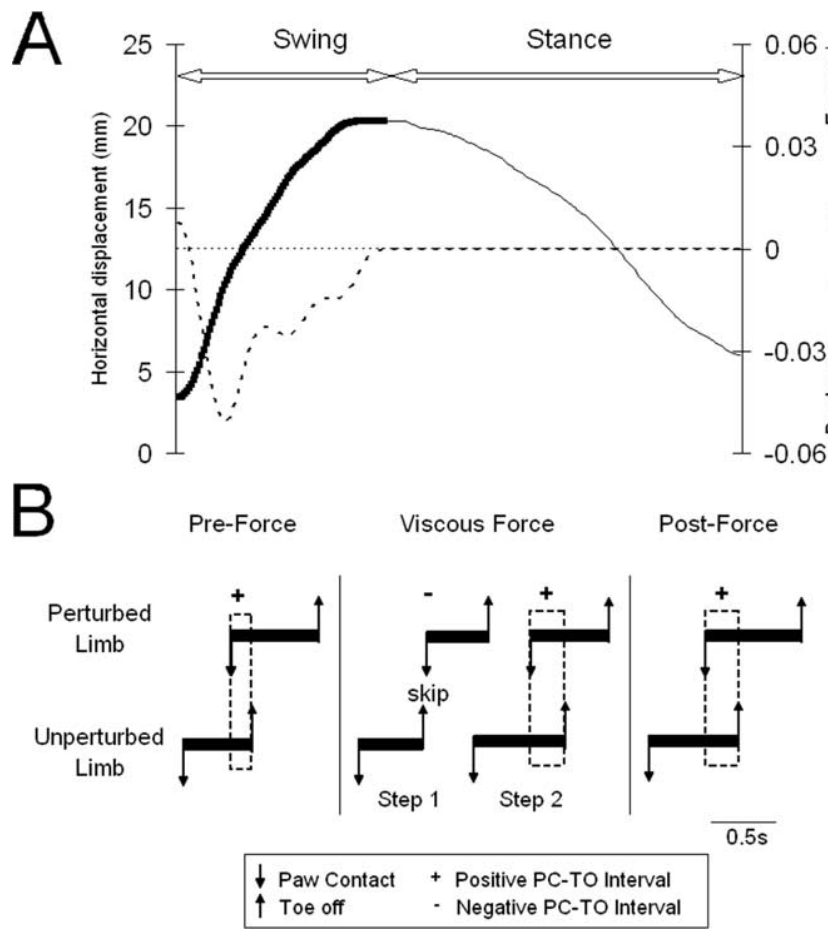

Figure 1. Examples of the robotic-applied viscous force and its effects on PC-T0 interval. $A$, Horizontal displacement (black line) of the ankle and the viscous force (dashed line) delivered to the ankle during one step cycle. Thick and thin black lines indicate swing and stance, respectively. B, Hindlimb coordination before (Pre-Force), during (Viscous Force), and after (PostForce) exposure to the unilateral viscous force in one representative ST rat. Note that the first steps during the Pre-force and Post-force conditions are shown, whereas the first two steps during the Viscous Force are shown. The horizontal black lines represent the stance phases. Downward arrows and upward arrows represent $\mathrm{PC}$ and $\mathrm{TO}$, respectively. Positive and negative $\mathrm{PC}-\mathrm{TO}$ intervals are denoted by + and - signs, respectively. Rectangles indicate the duration of the interval between $\mathrm{PC}$ of the perturbed limb and $\mathrm{TO}$ of the unperturbed limb during step cycles for positive PC-T0 intervals. "Skip" denotes that both hindpaws were airborne.

\section{Results}

\section{The robotic device delivered a unilateral viscous force that perturbed hindlimb coordination}

We used a robotic device to deliver a velocity-dependent force that opposed only forward movement in the ankle of one hindlimb while the ST rats stepped on a treadmill. Figure $1 A$ shows a single step cycle trajectory recorded by the robotic device during exposure to the viscous force. As the ankle moved forward during the swing phase of stepping, the robotic device delivered a backward-directed force that resisted forward motion of the ankle (Fig. 1A, Swing, dashed line). The average peak backward force applied during swing was $0.03 \pm 0.007 \mathrm{~N}$ ( $2 \%$ body weight). When the ankle moved backward during stance, no forces were applied (Fig. $1 A$, Stance, dashed line).

To determine whether ST rats adjusted hindlimb coordination to the unilateral viscous force, we first recorded ankle movements during hindlimb stepping for 10 step cycles without any force applied to the hindlimb. Immediately after the last preforce step, the unilateral viscous force was activated, and we recorded ankle movements during 10 perturbed step cycles. After the last perturbed step, the viscous force was inactivated, and the rats performed an additional 10 steps in the absence of the force. Figure $1 B$ illustrates the coordination between the hindlimbs of one ST rat during a locomotor perturbation experiment. We used the interval between the PC in the perturbed hindlimb and the TO in the unperturbed hindlimb as a simple measure of hindlimb 
coordination. During preforce stepping, $\mathrm{PC}$ in the perturbed hindlimb occurred before TO in the unperturbed hindlimb, and we denote this sequence as a positive PC-TO interval (Fig. $1 B$, Pre-Force). This is the expected pattern of hindlimb coordination during slow walking, and this ensures that swing in one hindlimb does not occur until the opposite hindlimb has begun stance (Cruse and Warnecke, 1992). However, when the unilateral viscous force was activated, the PC-TO interval shortened. In many instances, a reversal of the PC and TO events was observed. This meant the unperturbed hindlimb began swing before the perturbed hindlimb initiated stance. Thus, both hindpaws were briefly airborne giving the appearance of "skipping." We denote this sequence as a negative PC-TO interval (Fig. $1 B$, Viscous Force, Step 1). A positive PC-TO interval returned after repetitive exposure to the viscous force (Fig. $1 \mathrm{~B}$, Viscous Force, Step 2 ). When the viscous force was inactivated, the $\mathrm{PC}-\mathrm{TO}$ interval remained positive (Fig. $1 B$, Post-Force).

\section{ST rats corrected errors in hindlimb coordination after repetitive exposure to the unilateral viscous force}

Figure $2 \mathrm{~A}$ illustrates the changes in hindlimb coordination during exposure to the unilateral viscous force. Before the viscous force was activated, the average PC-TO interval generated by the ST rats was $0.10 \pm$ $0.02 \mathrm{~s}$ (Fig. $2 \mathrm{~A}$, dashed line). When the viscous force was applied to the ankle, the mean PC-TO interval for the first perturbed step was $-0.05 \pm 0.02 \mathrm{~s}$. The negative value for the PC-TO interval indicated that the unperturbed hindlimb initiated swing while the perturbed hindlimb was still executing swing (i.e., both hindpaws were airborne). A negative mean PC-TO interval was also found for perturbed steps 2 and 3 (Fig. 2A, Steps 2 and 3). The mean PC-TO interval increased after the first perturbed step and reached the preforce average after the fifth perturbed step (Fig. $2 A$, Steps 2-5). Based on analyses using a repeated-measures ANOVA and Bonferroni post hoc tests, the PC-TO value for the first perturbed step was significantly less than the mean preforce value (Fig. $2 A$, compare Step 1 with preforce average). No other significant changes were found.

To determine whether step-to-step variability in the PC-TO interval could have produced the observed improvements in hindlimb coordination, we also performed the experiments under a null force condition (horizontal viscous field coefficient, $B_{x x}$ $=0$ ). Figure $2 B$ illustrates the hindlimb coordination of ST rats during the null force testing. No significant difference was found between the mean PC-TO interval for any of the steps and the preforce average (Fig. 2B, Steps 1-10). However, there was a significant difference between the mean PC-TO interval in the first step of the null condition and the mean PC-TO interval in the first step of testing with the unilateral viscous force (compare Fig. 2A,B, Step 1). This further indicates a strong effect of the unilateral viscous force on the first perturbed step.

We next examined whether the perturbed and/or unper-
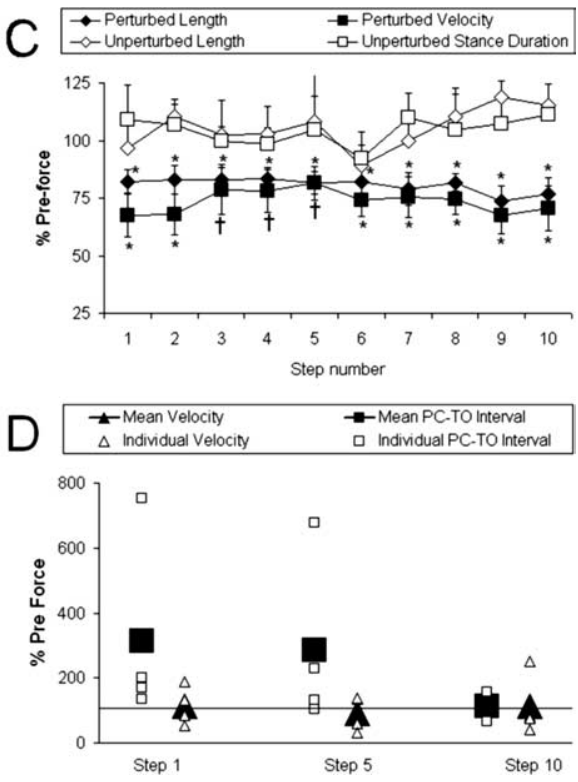

Figure 2. Step cycle characteristics during the viscous and null force tests. $\boldsymbol{A}$, Plot of the mean PC-TO interval during the application of the unilateral viscous force (filled diamonds). $\boldsymbol{B}$, Plot of the mean PC-T0 interval during the null force condition (filled diamonds). In $\boldsymbol{A}$ and $\boldsymbol{B}$, the dashed line is the mean PC-TO interval during the preforce stepping. $\boldsymbol{C}$, Plot of the mean step (filled triangles) and mean PC-T0 interval (filled squares) of the perturbed limb after the viscous force was 作

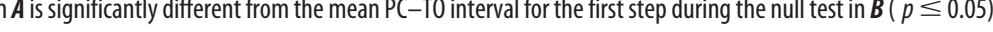
In $C^{*}$ and $†$ indicate that only the values for the step length and velocity of the perturbed hindlimb are significantly different from and $p \leq 0.05$, respectively.

turbed hindlimbs adapted to the viscous force. One possibility was that the perturbed hindlimb overcame the opposing force. We found that when the force was activated, the mean step length and mean forward velocity in the perturbed hindlimb significantly decreased (Fig. 2C, Step 1, filled diamonds and squares). This significant reduction was maintained while the force was applied (Fig. 2C, Steps 2-10, filled diamonds and squares). Thus, hindlimb coordination improved without recovery of the preforce length or velocity in the perturbed hindlimb.

Another possibility was that the unperturbed hindlimb adapted to the force by delaying the onset of swing. This would allow time for PC in the perturbed hindlimb. If so, then a prolonged stance phase in the unperturbed hindlimb would be expected. Although some rats adapted to the viscous force by generating a longer stance phase (Fig. $1 B$, Viscous Force, stance in Step 2), the average stance duration and step length in the unperturbed hindlimb was not significantly increased when the viscous force was activated (Fig. 2C, open diamonds and squares).

To test for the presence of aftereffects, the viscous force was inactivated after the 10th perturbed step, and we recorded stepping for an additional 10 steps. If indeed there was an aftereffect, then we would expect that hindlimb coordination would change in a manner that was opposite to the coordination errors caused by the viscous force. Specifically, a positive PC-TO interval that was larger than the preforce PC-TO interval should occur when the force was removed. Only four of the rats were able to maintain consistent stepping during the postforce condition. However, an aftereffect in the PC-TO interval was observed in each of these 
four rats (Fig. 2D, Step 1, open squares). The mean PC-TO interval for the first step after the removal of the viscous force was $315 \%$ of the preforce average (Fig. 2D, Step 1). By the 5 th and 10th steps, the mean PC-TO interval decreased to 285 and $113 \%$ of the preforce average, respectively (Fig. 2D, Steps 5 and 10). These changes, however, were not statistically significant. No significant aftereffect in forward velocity of the hindpaw was found (Fig. 2D, triangles).

Errors in hindlimb coordination were related to prolonged hang time in the perturbed hindpaw

To begin to understand how the unilateral viscous force disrupted hindlimb coordination, we examined the relationship between the PC-TO interval and the time that the perturbed hindpaw was suspended in the air during swing. The decision to study this relationship was based on the observation that when the unilateral viscous force was activated, forward movement of the perturbed hindpaw slowed down considerably such that the perturbed hindpaw appeared to hang in the air for an extended period of time.

Figure 3 illustrates the hang phenomena for one representative ST rat. The period of time when little vertical movement occurred in the paw (i.e., vertical velocity close to 0 ) before the initiation of PC is highlighted in Figure $3 A-C$ (Vertical Velocity plots, thick line). We measured this period of time, which we call hang time, before and after the unilateral force was activated. We found that mean hang time during the first perturbed step $(0.34 \pm 0.05 \mathrm{~s})$ was significantly greater than the preforce average $(0.16 \pm 0.04 \mathrm{~s} ; p \leq 0.01)$ (Fig. $3 A, B)$. However, hang time improved after the $S T$ rats had been exposed to the unilateral viscous force. Specifically, the mean hang time of the sixth perturbed step $(0.16 \pm 0.03 \mathrm{~s})$ recovered to the preforce average (Fig. $3 A, C)$. This means that the ST rats adapted to the unilateral viscous force by initiating PC sooner, thereby terminating swing.

Figure $3 D$ illustrates the relationship between hang time in the perturbed hindlimb and the PC-TO interval. The data collected during all perturbed steps from all of the ST rats were used to generate this plot. If the prolonged hang time in the perturbed limb was related to the errors in hindlimb coordination, then negative PC-TO intervals would be expected to occur with large hang time values. We would also expect that positive $\mathrm{PC}-\mathrm{TO}$ intervals would tend to occur with short hang times. We found that, indeed, hang time and PC-TO interval were negatively correlated and that this correlation was highly significant (Pearson correlation, $-0.328 ; p \leq 0.001)$.

\section{Discussion}

Adjustments in hindlimb coordination are acquired through experience

One role that the spinal cord plays in producing locomotor adaptations is to generate fast responses to perturbations. However, some adaptations in stepping are acquired through practice. For these adaptations, movement errors are made before the necessary adjustments are developed. We found that the ST rats were unable to adjust hindlimb coordination patterns during the first perturbed step cycle, but rather, hindlimb coordination improved when stepping had been perturbed for two to five step cycles. These findings indicated that, in addition to generating fast corrective responses, the lumbar spinal cord generated some adjustments in hindlimb stepping patterns through experience.

To date, there has been little evidence that the spinal cord contributed more than rapid responses during locomotor adaptations. Perhaps the best known evidence came from a pilot study
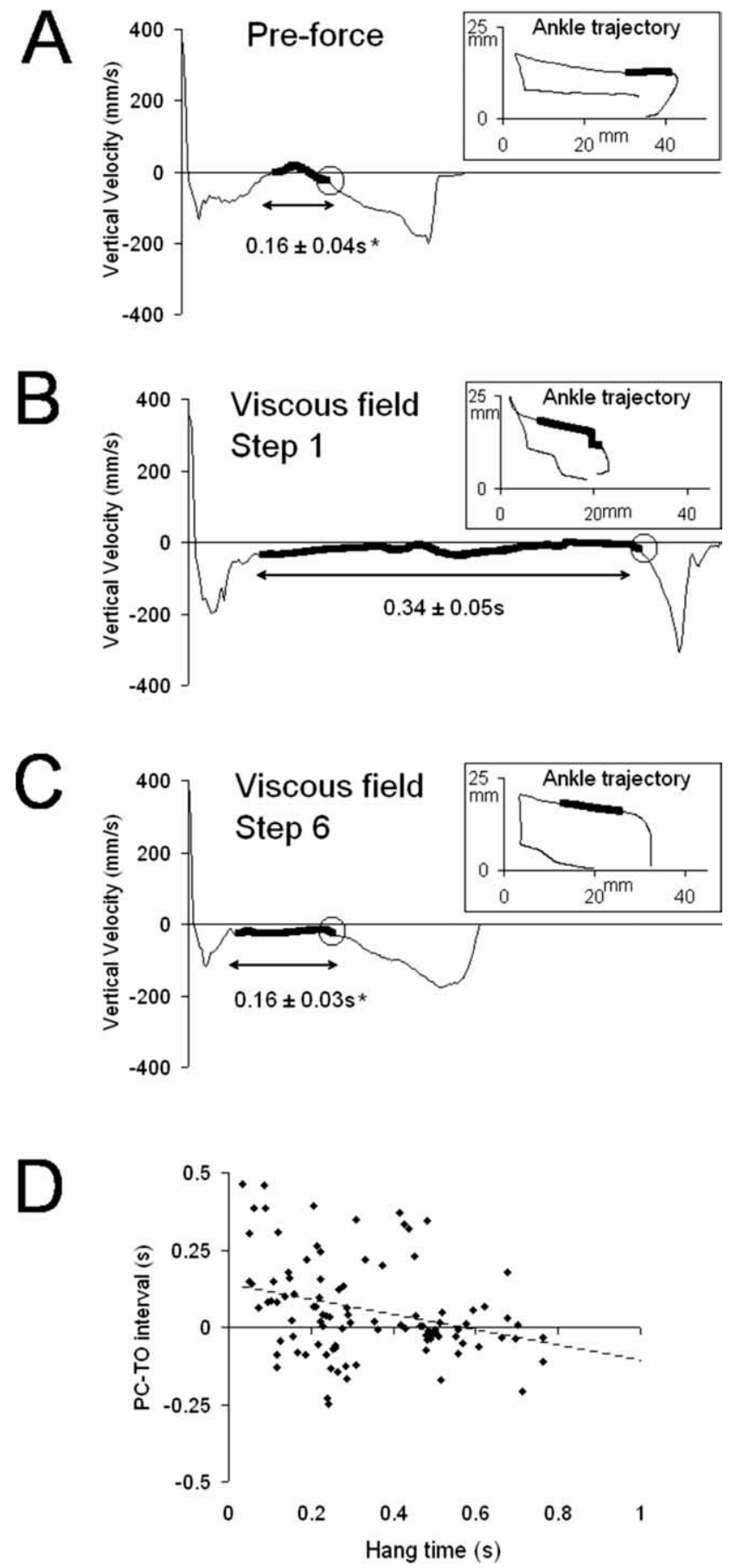

Figure 3. Relationship between hang time in the perturbed limb and PC-TO interval. $A$, Plot of the vertical velocity over time from a single step cycle recorded during preforce testing. $\boldsymbol{B}$, Plot of the vertical velocity over time recorded during the first perturbed step. $C$, Plot of the vertical velocity over time recorded during the sixth perturbed step. The data shown are from one representative ST rat. The insets show the corresponding ankle trajectory. The thick black line indicates hang time. Circles in the velocity plots indicate the beginning of downward movement in the hindpaw before $P C$. The mean hang times \pm SE are shown below the velocity plots $(n=$ 10). The asterisk indicates a significant difference with the mean hang time for the viscous field, Step 1 condition $(p \leq 0.01)$. D, Plot of the PC-T0 interval versus hang time in the perturbed hindlimb. Data shown are from all of the perturbed steps in all ST rats (10 steps for each rat; $n=$ 10 rats). The Pearson correlation (coefficient, -0.328 ) was significant at the level of $p<0.01$.

that showed an ST cat learned to avoid an obstacle that blocked the forward movement of the hindpaw (Hodgson et al., 1994). After the initial steps were perturbed, the ST cat adjusted hindlimb trajectories, and during some step cycles, the hindpaw was 
completely lifted over the obstacle. Unfortunately, the experiment did not control for inherent step-to-step variability. In the present study, we dealt with the potentially confounding factor of stepping variability by including a null force test. After comparing the data from the force and null force tests, we conclude that it is unlikely that the variability in stepping patterns could account for either the errors or the subsequent improvements in coordination.

We found that although hindlimb coordination improved with experience, step length and forward velocity failed to recover even after repetitive exposure to the perturbation. There is evidence from two recent studies that able-bodied human subjects learned to adapt to viscous forces applied to their legs by overcoming the opposing force and regaining some normal step cycle characteristics (Emken and Reinkensmeyer, 2005; Lam et al., 2006). In the present study, the peak force applied to the hindlimb may have exceeded the amount of muscle force generated by ST rats. The inability to recover normal characteristics may also indicate that although some corrections in movement (i.e., hindlimb coordination) can be learned by the lumbar spinal cord, other adjustments in step cycle characteristics may require supraspinal structures such as the cerebellum (Morton and Bastian, 2006).

\section{Possible mechanisms for spinally mediated corrections in coordination}

Based on the present findings, it appears that the adaptations to the viscous force involved adjustments in the swing phase of the perturbed hindlimb (Fig. 3). Interestingly, adjustments in the unperturbed hindlimb that could have facilitated coordination (i.e., prolonged stance phase) were not consistently observed (Fig. 2C). One possibility is that hip-related sensory information influenced the swing-to-stance transition (McVea et al., 2005) in the perturbed hindlimb. However, we did not perform kinematic analyses of hindlimb joint movement, so the contribution of hiprelated or other proprioceptive information is unclear.

Did the spinal cord use a feedforward strategy to correct hindlimb coordination? Human subjects use feedforward strategies when they adapt their stepping patterns to external perturbations (Emken and Reinkensmeyer, 2005; Lam et al., 2006; Morton and Bastian, 2006), and perhaps feedforward strategies were also used by the ST rats in the present study. This is an intriguing idea because it would suggest that the lumbar spinal cord corrected movement errors by anticipating the effects of the viscous forces acting on the hindlimbs. One key characteristic of feedforward strategies is the presence of aftereffects that occur after the perturbation is removed (Shadmehr and Mussa-Ivaldi, 1994). Only one previous study has provided evidence for a spinally mediated aftereffect, and that was the pilot study of an ST cat stepping over an obstacle (Hodgson et al., 1994). We observed aftereffects when the viscous force was inactivated. Specifically, a large PC-TO interval was found in four ST rats during the postforce testing. Interestingly, the other six ST rats stumbled when the viscous force was inactivated. This raises the possibility that the stumbling response itself constituted a type of aftereffect on hindlimb coordination. Clearly, additional studies are necessary before we can determine whether the spinal cord has the capacity to perform feedforward processing during locomotor adaptations.

The present results have potential implications for gait training strategies after spinal cord injury. Based on the present findings, the spinal cord is capable of modulating locomotor activity in response to asymmetric viscous loading during stepping. Studies of long-term gait training are necessary to determine whether applying forces to one limb will provide an optimal stimulus for plasticity within the locomotor-generating spinal circuitry.

\section{References}

Cha J, Heng C, Reinkensmeyer DJ, Roy RR, Edgerton VR, de Leon RD (2007) Locomotor ability in spinal rats is dependent on the amount of activity imposed on the hindlimbs during treadmill training. J Neurotrauma 24:1000-1012.

Cruse H, Warnecke H (1992) Coordination of the legs of a slow-walking cat. Exp Brain Res 89:147-156.

Emken JL, Reinkensmeyer DJ (2005) Robot-enhanced motor learning: accelerating internal model formation during locomotion by transient dynamic amplification. IEEE Trans Neural Syst Rehabil Eng 13:33-39.

Forssberg H (1979) Stumbling corrective reaction: a phase-dependent compensatory reaction during locomotion. J Neurophysiol 42:936-953.

Hodgson JA, Roy RR, de Leon R, Dobkin B, Edgerton VR (1994) Can the mammalian lumbar spinal cord learn a motor task? Med Sci Sports Exerc 26:1491-1497.

Lam T, Anderschitz M, Dietz V (2006) Contribution of feedback and feedforward strategies to locomotor adaptations. J Neurophysiol 95:766-773.

McVea DA, Donelan JM, Tachibana A, Pearson KG (2005) A role for hip position in initiating the swing-to-stance transition in walking cats. J Neurophysiol 94:3497-3508.

Morton SM, Bastian AJ (2006) Cerebellar contributions to locomotor adaptations during splitbelt treadmill walking. J Neurosci 26:9107-9116.

Shadmehr R, Mussa-Ivaldi FA (1994) Adaptive representation of dynamics during learning of a motor task. J Neurosci 14:3208-3224.

Timoszyk WK, de Leon RD, London N, Roy RR, Edgerton VR, Reinkensmeyer DJ (2002) The rat lumbosacral spinal cord adapts to robotic loading applied during stance. J Neurophysiol 88:3108-3117. 